\title{
Diagnostic value of serum procalcitonin for diagnosis of bacterial infection in patients with chronic kidney disease under hemodialysis
}

\author{
Rasoul Estakhri ${ }^{1,2}$, Nava Moghadasian Niaki ${ }^{3 *(\infty}$, Hamid Noshad ${ }^{4}$, Mohammad Asghari $^{5}$, Hojjat Barghi ${ }^{(\circledR)}$ \\ ${ }^{1}$ Kidney Research Center,Tabriz University of Medical Sciences, Tabriz, Iran \\ ${ }^{2}$ Department of Pathology, Sina Training and Treatment Center, Tabriz, Iran \\ ${ }^{3}$ Department of Pathology, Imam Reza Training and Treatment Center, Tabriz, Iran \\ ${ }^{4}$ Department of Nephrology, Sina Training and Treatment Center, Tabriz, Iran \\ ${ }^{5}$ Department of Epidemiology, Health and Neutrition Faculty, Tabriz, Iran
}

*Correspondence to

Nava Moghadasian Niaki,

Email: Moghaddasiann@

tbzmed.ac.ir

Received 14 Nov. 2019 Accepted 25 Dec. 2019 Published online 6 Jan. 2020

Keywords: Procalcitonin Hemodialysis, Bacterial infection, Chronic kidney disease, End-stage renal diseases, C-reactive protein

\begin{abstract}
Introduction: Bacterial infections are common causes of mortality and morbidity among chronic kidney disease (CKD) patients under hemodialysis.

Objectives: In this study the diagnostic value of serum procalcitonin for diagnosis of bacterial infections in patients with CKD under hemodialysis was assessed.

Patients and Methods: In this cross-sectional comparative investigation, 47 patients with CKD under hemodialysis were enrolled to the study. We studied the relationship of serum procalcitonin (PTC) and C-reactive protein (CRP) levels (before and after dialysis) with "positive bacterial culture" and "systemic inflammatory response syndrome $(\mathrm{SIRS})^{\prime \prime}$ results. Sensitivity and specificity were determined by ROC test.

Results: Serum PTC before and after dialysis as well as the CRP before dialysis had no significant association with positive bacterial culture $(P=0.492, P=0.1$ and $P=0.268$ respectively), however after-dialysis CRP had a significant association with positive bacterial culture $(P=0.032)$.

Conclusion: According to the obtained results, it may be concluded that the diagnostic value of serum PTC for diagnosis of positive culture bacterial infections in hemodialysis patients is not satisfactory since the serum CRP level especially after dialysis is more useful.
\end{abstract}

\section{Introduction}

Chronic kidney disease (CKD) is seen in ten percent of Americans adults $(1,2)$. Hemodialysis is the main dialysis method with $23 \%$ mortality rate, since among them infection is the main cause of death (3). The relative risk of mortality in hospitalized hemodialysis patients with bacteremia and septicemia, in first six months of starting the dialysis is seven times more versus non-hospitalized subjects (4), with even higher hospital admission for septicemia in hemodialysis versus peritoneal dialysis cases (5). End-stage renal disease cases are susceptible to systemic bacteremia with worse prognosis and more complications (68). Patients under hemodialysis are susceptible to catheter and blood infections (9). The symptoms of infection in these patients are usually mild and non-specific since, the usual laboratory markers are affected by uremic status such as leukocyte count, C-reactive protein (CRP), erythrocyte sedimentation rate (ESR), and interleukins including interleukin

\section{Key point}

In a study on 47 patients with CKD under hemodialysis, we found that serum C-reactive protein had better applicability versus procalcitonin and the increase in C-reactive protein had a better predictive value.

6 (IL-6), IL-1b, and also TNF-alpha $(10,11)$. CRP is a sensitive non-specific index for infection (12), since ESR and leukocyte (WBC) count, are not useful to differentiate infectious and non-infectious etiologies (13). Additionally, microbiological cultures may be slow and non-sensitive $(14,15)$. Procalcitonin (PCT) is currently introduced with half-life of 25-30 hours showing infection in serum levels over $0.5 \mathrm{ng} / \mathrm{mL}$ (16). It increased in patients with bacterial and fungal infections that have normal renal function (17). Despite other markers, it is not increased in viral and local infections and also non-infectious inflammations $(18,19)$. PCT may be removed by renal pathway due

\footnotetext{
Copyright (C) 2020 The Author(s); Published by Nickan Research Institute. This is an open-access article distributed under the terms of the Creative Commons Attribution License (http://creativecommons.org/licenses/by/4.0), which permits unrestricted use, distribution, and reproduction in any medium, provided the original work is properly cited.
} 
to small molecular size (20). However, it is not the main metabolic route (21); it may be increased in CKD cases $(22,23)$. Regarding the interaction between PCT level with vitamin $\mathrm{D}$, aluminum toxicity, beta 2 microglobulin and secondary hyperparathyroidism, these parameters should be considered in metabolic assessment (24). High rate of bacterial infections in CKD cases may make difficult differentiation between non-infectious inflammations (25), antibiotic resistance, and drug hypersensitivity in cases under empirical antibiotic therapy (26-29). This condition led to a need for further accurate methods in hemodialysis patients $(30,31)$.

\section{Objectives}

In this study the diagnostic value of serum PTC for diagnosis of bacterial infections in patients with CKD under hemodialysis was assessed.

\section{Patients and Methods}

\section{Study design}

In this cross-sectional comparative study, 47 patients with CKD under high flux hemodialysis three times weekly (each time 4 hours) in hemodialysis sections of two hospitals in Tabriz, Iran in 2018 were enrolled. Inclusion criteria were age older than 18 years, CKD, suspicion of bacterial infections (according to weakness, fever, chills after dialysis in last two weeks, painful or inflamed fistula, discharge, redness and warmness at catheter site, upper/ lower respiratory tract infections, upper/lower urinary tract infection, skin and soft tissue infection, diabetic ulcer, peritonitis, intra-abdominal wound, other infection foci, radiological/ultrasound evidences for infectious focus, and minimally two systemic inflammatory response syndrome (SIRS) criteria (32). Exclusion criteria were rheumatologic dieses/malignancy, current surgery, burn in last 4 weeks, antibiotic therapy longer than 48 hours and we also excluded all patients with remarkable malnutrition according to serum albumin, calcium, phosphorus and muscular mass loss.

Microbial culture (done for blood samples up to three times), CRP, PCT, urea, and creatinine were assessed to determine the dialysis adequacy. Additionally, CBC, serum calcium, and phosphorous were assessed. The serum PTC, CBC, and CRP levels were compared between those with and without positive cultures while sensitivity and specificity were determined by ROC test. We corrected PCT values for all patients during hemodialysis according to amount of ultrafiltration (volume reduction). Besides, the other clinical, laboratory and demographic factors were evaluated and recorded in the checklists.

The CBC was assessed with Sysmex, Japan device by impedance electricity. The CRP was measured by turbidimetric method (Pars Azmoon, Iran kit) and by Mindry device, China. For PCT the Rosh, AmericanGermany kits were used.

\section{Ethical issues}

Human rights were respected in accordance with the Helsinki Declaration 1975, as revised in 1983. The ethical committee of Tabriz University of Medical Sciences (Ethical code; IR. TBZMED.IR.1395.204) approved the study. The informed consents were taken from the patients. Besides, this study was extracted from the M.D, thesis conducted by Nava Moghadasian Niaki (Thesis\# 94.3-6.10) at this University.

\section{Statistical analysis}

Data analysis was finally done by STATA14 software. The utilized test was ROC and area under the curve (AUC) was calculated for CRP and PTC with appropriate sensitivity and specificity. In addition, Wilcoxon and $t$ tests were utilized. $P$ values less than 0.05 were considered significant.

\section{Results}

In this study, the mean age was $62.2 \pm 17.5$ years, with 23 males and 24 females. The mean BMI (body mass index) was $26.5 \pm 5 \mathrm{~kg} / \mathrm{m}^{2}$. In 11 cases, there was a history of intensive care unit (ICU) admission. Catheter and fistula were seen in $89.4 \%$ and $12.7 \%$, respectively. Catheter was in jugular vein, subclavian, and femoral in 54.8\%, 19\% and $26.2 \%$ of patients, respectively. In addition, the fistula infection was present in $16.7 \%$.

\section{Para-clinical Data}

The culture was positive in 20 cases $(42.6 \%$ including 9 cases of blood culture, 10 patients with urine culture, 1 catheter culture, 1 wound culture, 1 ascites culture, and 1 with synovial fluid culture). Gram staining was positive in $77.8 \%$. Urine culture was positive in $21.3 \%$ that was grampositive in $10 \%$. The microbial germs were pseudomonas, E. coli, Klebsiella, enterococci, Staphylococcus aureus, Staphylococcus epidermidis, and streptococci in 1, 10, 4, 3, 3,4 , and 1 case. The results of cell count and biochemical tests are shown in Table 1.

As shown in Table 2, the serum levels of PCT and CRP had no significant difference before and after dialysis $(P>0.05)$. The laboratory measurements in variables are shown in Table 3 while among them only hemoglobin and ionized calcium were significantly different.

\section{Data analysis according to culture results}

The PTC level before and after dialysis was not related to infection $(P=0.492$ and 0.1$)$. Additionally no significant association was found for CRP and infection before dialysis. However, post-dialysis CRP was significantly related to infection $(P=0.032)$ with AUC of 0.684 and sensitivity and specificity of $80 \%$ and $70.4 \%$ with cut-off 66. AUC for WBC and PMN was 69.99\% (Figure 1).

As shown in Table 4, PCT before and after dialysis and CRP before dialysis were not related to culture results $(P>0.05)$; serum CRP after dialysis had a significant difference between culture positive and culture negative 
Table 1. Cell count and biochemical tests in patients

\begin{tabular}{|c|c|c|c|c|c|}
\hline Factor & Mean & Median & Standard Deviation & Minimum & Maximum \\
\hline WBC (count/mL) & 13544.04 & 10900.00 & 6697.19 & 4500.00 & 36200.00 \\
\hline Neutrophil (\%) & 80.12 & 83.65 & 13.77 & 13.20 & 96.30 \\
\hline Lymphocyte (\%) & 14.20 & 10.90 & 13.03 & 2.70 & 85.80 \\
\hline Hemoglobin (g/dL) & 9.82 & 9.50 & 1.62 & 6.00 & 13.40 \\
\hline $\operatorname{MCV}(\mathrm{fl})$ & 88.03 & 87.70 & 7.52 & 62.00 & 106.00 \\
\hline Platelet (count/mL) & 225000 & 2040 & 91732 & 65000 & 436000 \\
\hline Albumin $(\mathrm{g} / \mathrm{dl})$ & 3.12 & 3.30 & 0.72 & 1.30 & 4.10 \\
\hline Total calcium (mg/dL) & 8.19 & 8.20 & 1.12 & 4.80 & 11.60 \\
\hline Ionized calcium (mg/dL) & 1.02 & 1.01 & 0.15 & 0.72 & 1.58 \\
\hline Phosphorous (mg/dL) & 5.27 & 4.80 & 2.36 & 1.40 & 12.30 \\
\hline
\end{tabular}

Table 2. Renal and inflammatory markers before and after dialysis

\begin{tabular}{|c|c|c|c|c|c|}
\hline Factor & Mean & Median & Standard Deviation & Minimum & Maximum \\
\hline Urea-before $(\mathrm{mg} / \mathrm{dL})$ & 138.95 & 120.00 & 64.55 & 48.00 & 325.00 \\
\hline Urea-after (mg/dL) & 94.27 & 90.00 & 48.30 & 33.00 & 267.00 \\
\hline Cr-before (mg/dL) & 6.77 & 5.87 & 3.19 & 3.00 & 16.13 \\
\hline Cr-after (mg/dL) & 4.96 & 4.58 & 2.21 & 1.83 & 11.80 \\
\hline PCT-before $(\mathrm{ng} / \mathrm{mL})$ & 2.72 & 1.18 & 4.52 & .05 & 24.75 \\
\hline PCT-after (ng/mL) & 3.48 & 1.25 & 6.91 & .04 & 33.00 \\
\hline CRP-before $(\mathrm{mg} / \mathrm{L})$ & 73.37 & 67.00 & 35.27 & 2.20 & 136.30 \\
\hline CRP-after (mg/L) & 74.95 & 67.00 & 36.49 & 2.80 & 148.00 \\
\hline
\end{tabular}

patients $(P=0.032)$. Serum PCT and CRP were not differed according to mortality in patients $(P>0.05)$. Serum PCT before and after dialysis and CRP before dialysis were not related to ICU admission $(P>0.05)$; however, after dialysis, CRP levels were significantly higher in ICU admitted cases $(P=0.044)$.

Data analysis according to SIRS results As shown in Figure 2, the WBC, PMN, lymphocyte and platelet were significantly differed between positive and negative SIRS groups $(P<0.05)$ with AUC of $100 \%, 97.5 \%$,

\section{ROC Curve}

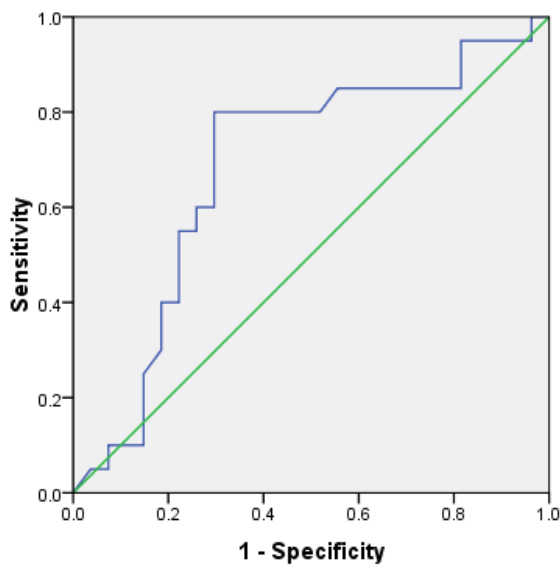

Diagonal segments are produced by ties.

Figure 1. ROC curve for after-dialysis CRP.
$100 \%$, and $95 \%$, respectively. Moreover, as demonstrated in Figure 3 and Table 5, the initial PCT $(P=0.011)$ and final PCT $(P=0.003)$ and the final CRP $(P=0.004)$ had significant difference according to SIRS with AUC of $72.5 \%, 76.4 \%$, and $75.9 \%$, respectively.

Discussion

In this study, the diagnostic value of serum PTC for diagnosis of bacterial infections in patients with CKD under hemodialysis was assessed. In this study, the base for comparison was conducted once by culture and once by SIRS. For culture comparison, the PCT before dialysis had AUC of $56 \%$ and after dialysis, it was $65 \%$. It was $60 \%$ and $68 \%$ for CRP before and after dialysis, respectively. This study showed lower predictive value of PCT versus CRP according to culture. When classification of patients to infectious and non-infectious groups was conducted by SIRS criteria, better diagnostic values of PCT and CRP were obtained. The possible cause of this difference was presence of slow-growing microorganisms or false positive/negative results. Additionally, the higher cut-off point for PCT and CRP versus the general population should be considered for data interpretation in dialysis patients.

El-Sayed et al (30) reported that PT had sensitivity and specificity of $80 \%$ and $35 \%$ with cut-off point of 0.5 . Similarly, they reported that PCT had no good sensitivity and specificity like our study when comparing by culture. Lee et al (28) reported, a significantly higher serum PCT in infection cases. They considered cut-off point of 0.75 
Table 3. Laboratory markers in cases with positive and negative culture

\begin{tabular}{|c|c|c|c|}
\hline Variable & Culture & Mean & Standard Deviation \\
\hline \multirow{2}{*}{ Age $(y)$} & Positive & 68.1500 & 17.10732 \\
\hline & Negative & 57.8148 & 16.82955 \\
\hline \multirow{2}{*}{ WBC (count/mL) } & Positive & 15400.0000 & 5906.90942 \\
\hline & Negative & 12169.2593 & 7017.42169 \\
\hline \multirow{2}{*}{$\mathrm{PMN} \%$} & Positive & 84.1600 & 10.12492 \\
\hline & Negative & 77.0154 & 15.50478 \\
\hline \multirow{2}{*}{ Lymphocyte\% } & Positive & 10.2650 & 7.59226 \\
\hline & Negative & 17.2415 & 15.49021 \\
\hline \multirow{2}{*}{$\mathrm{Hb}(\mathrm{g} / \mathrm{dl})$} & Positive & 10.3650 & 1.58056 \\
\hline & Negative & 9.4077 & 1.55356 \\
\hline \multirow{2}{*}{$\mathrm{MCV}(\mathrm{fl})$} & Positive & 86.9842 & 8.27864 \\
\hline & Negative & 88.8000 & 6.98478 \\
\hline \multirow{2}{*}{ PLT (count/mL) } & Positive & 233150.0000 & 100021.19512 \\
\hline & Negative & 218480.0000 & 86066.89259 \\
\hline \multirow{2}{*}{ Urea before (mg/dL) } & Positive & 143.2000 & 67.58823 \\
\hline & Negative & 135.8148 & 63.33005 \\
\hline \multirow{2}{*}{ Urea after (mg/dL) } & Positive & 97.9500 & 47.16765 \\
\hline & Negative & 91.5556 & 49.84695 \\
\hline \multirow{2}{*}{ Cr before $(\mathrm{mg} / \mathrm{dL})$} & Positive & 6.7737 & 3.09715 \\
\hline & Negative & 6.7733 & 3.32262 \\
\hline \multirow{2}{*}{ Cr-after (mg/dL) } & Positive & 5.0184 & 2.39039 \\
\hline & Negative & 4.9237 & 2.13659 \\
\hline \multirow{2}{*}{ Alb (g/dL) } & Positive & 2.7857 & .77552 \\
\hline & Negative & 3.3250 & .64544 \\
\hline \multirow{2}{*}{ Ca total $(\mathrm{mg} / \mathrm{dL})$} & Positive & 8.4000 & 1.06829 \\
\hline & Negative & 8.0667 & 1.15392 \\
\hline \multirow{2}{*}{$\begin{array}{l}\text { lonized calcium (mg/ } \\
\mathrm{dL} \text { ) }\end{array}$} & Positive & 1.0873 & .19285 \\
\hline & Negative & .9870 & .11138 \\
\hline \multirow{2}{*}{ Phosphorus (mg/dl) } & Positive & 5.4500 & 2.55671 \\
\hline & Negative & 5.1556 & 2.26806 \\
\hline \multirow{2}{*}{ BMI (kg/m²) } & Positive & 26.5466 & 5.58320 \\
\hline & Negative & 26.5275 & 4.71329 \\
\hline \multirow{2}{*}{$\operatorname{GFR}\left(\mathrm{mL} / \mathrm{min} / 1.73 \mathrm{~m}^{2}\right)$} & Positive & 10.9168 & 4.61579 \\
\hline & Negative & 13.0243 & 5.23973 \\
\hline
\end{tabular}

Table 4. Serum PCT and CRP in positive and negative cultures

\begin{tabular}{lccc}
\hline Variable & Culture & Mean & Standard Deviation \\
\hline \multirow{2}{*}{ PCT before $(\mathrm{ng} / \mathrm{mL})$} & Positive & 3.38 & 6.13 \\
& Negative & 2.22 & 2.81 \\
\multirow{2}{*}{ PCT after $(\mathrm{ng} / \mathrm{mL})$} & Positive & 4.41 & 8.08 \\
& Negative & 2.77 & 5.95 \\
CRP before $(\mathrm{mg} / \mathrm{L})$ & Positive & 81.58 & 38.03 \\
& Negative & 67.30 & 32.47 \\
CRP after $(\mathrm{mg} / \mathrm{L})$ & Positive & 87.11 & 32.93 \\
& Negative & 65.95 & 36.95 \\
\hline
\end{tabular}

for PCT leading to sensitivity and specificity of $76 \%$ and $80 \%$. However, these findings were lower in our study according to culture that was improved according to SIRS. Accordingly, Herget-Rosenthal et al (30) reported sensitivity and specificity of 89 and 81 percent for cut-off point of 0.5 for PCT that was higher than CRP amounts of sensitivity and specificity in their study; however in our study CRP had better sensitivity and specificity in comparison to PCT, according to culture results.

Similar to our study, Steinbach et al (33) reported an

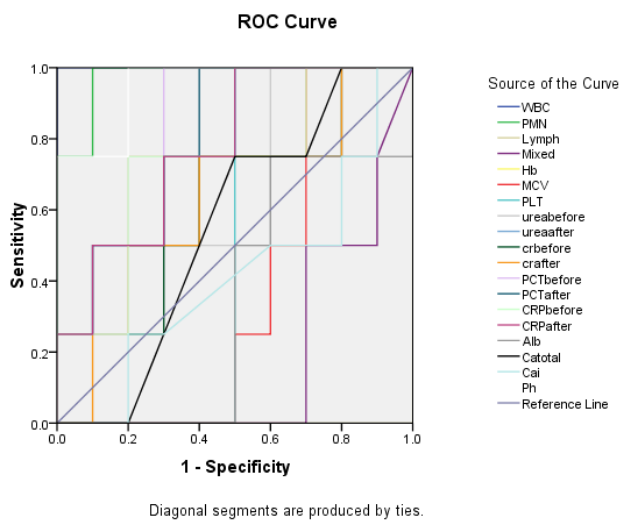

Figure 2. Comparison of variables according to SIRS.
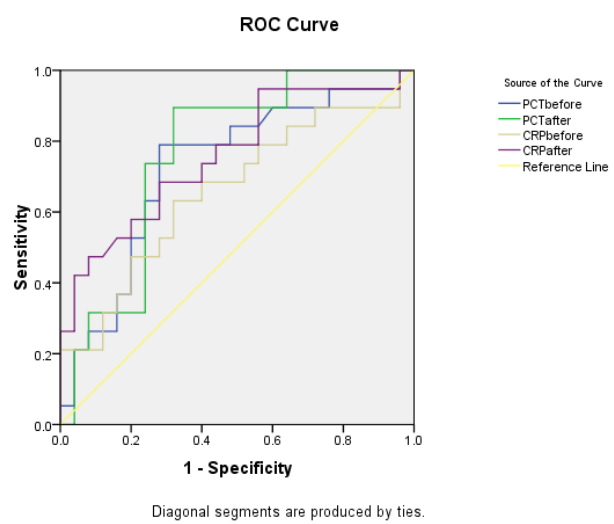

Figure 3. Comparison of PCT and CRP according to SIRS.

Table 5. PCT and CRP cut-off, sensitivity, and specificity according to SIRS

\begin{tabular}{llll}
\hline Variable & Cut-off & Sensitivity & Specificity \\
\hline PCT before $(\mathrm{ng} / \mathrm{mL})$ & 1.22 & $73.7 \%$ & $72 \%$ \\
PCT after $(\mathrm{ng} / \mathrm{mL})$ & 1.29 & $73.7 \%$ & $72 \%$ \\
CRP before $(\mathrm{mg} / \mathrm{L})$ & 66 & $66 \%$ & $63.2 \%$ \\
CRP after $(\mathrm{mg} / \mathrm{L})$ & 61.1 & $60 \%$ & $73.2 \%$ \\
\hline
\end{tabular}

increase in PCT and CRP levels with lower diagnostic value for PCT against CRP. Dumea et al (34) reported bacterial infection in 58 out of 82 patients showing with higher PCT in infection cases leading to sensitivity and specificity of 93 and 79 percent with cut off 0.5 . In our study, the PCT had not good results in comparison to culture results, but better results were seen when compared by SIRS. In the study by Fadel et al (35) among 102 patients of CKD with 34 culture positive cases showed sensitivity and specificity of $94 \%$ and $88 \%$ for PCT with a cut of point of 0.5 , respectively. These results were low in our study compared to the culture results, but improved by SIRS.

\section{Conclusion}

In our investigation, PCT has not acceptable diagnostic value in detection of bacterial infection in CKD patients on hemodialysis. Among the possible causes for these 
differences of results between our and previous studies, small sample size, lack of matching for ionized calcium (regarding the effect on calcitonin), and subdivision according to culture results (as gold standard of infection diagnosis), may be mentioned.

In the second phase of this study, our findings are also improved when we used SIRS results for evaluation of our data instead of culture results. It means that when we used the SIRS criteria as a base for comparison, better sensitivity and specificity were obtained with more similarity to other studies. This comparison of findings according to culture and SIRS results is distinguishing point of our study. Since our study was cross-sectional, the matching ability was decreased between groups. However, two groups were matched in majority of cases. The important causes of differed results between culture and SIRS were slow-growth of some organisms, simultaneous fungal or parasitic infections, human errors, non-reliable drug history especially for self-prescribed use of antibiotics leading to high-effect in PCT versus CRP when used in current 48 hours. Among other possible causes, sampling techniques and processing methods beside the pathogen microorganisms may be also considered when culturing the samples. These are effective on culture but the SIRS factors are less affected. In addition, it should be considered that this study was first time in Iran among stable hemodialysis patients. In addition, as an important point, we assessed both PCT and CRP markers while both culture and SIRS results were applied for comparison. We found that CRP had better applicability versus PCT and the increase in CRP had a better predictive value. However, the presence of some confounding factors needs to be considered in further studies.

\section{Limitation of the study}

The main limitation of our study is the low number of patients.

\section{Authors' contribution}

Design of the study and selecting the patients; RA, HN and NMN. Gathering the patients and data entering; NMN and HB. Data analysis; MA and NMN. Primary draft by RA and NMN. Final edition by RA, NMN and HB. All authors read and signed the paper manuscript.

\section{Conflicts of interest}

The authors declared that there was no conflict of interest.

\section{Ethical considerations}

Ethical issues including plagiarism, double publication, and redundancy have been completely observed by the authors.

\section{Funding/ Sources}

This research project is funded by Kidney Research Center, Tabriz University of Medical Sciences.

\section{References}

1. Laster M, Pereira RC, Salusky IB. Racial differences in bone histomorphometry in children and young adults treated with dialysis. J Bone. 2019;127:114-119. doi: 10.1016/j. bone.2019.06.004.

2. Hill NR, Fatoba ST, Oke JL, Hirst JA, O'Callaghan CA, Lasserson DS, et al. Global Prevalence of Chronic Kidney Disease - A Systematic Review and Meta-Analysis. PLoS One. 2016;11: e0158765. doi: 10.1371/journal.pone.0158765.

3. Viecelli AK, O'Lone E, Sautenet B, Craig JC, Tong A, Chemla $E$, et al. Vascular access outcomes reported in maintenance hemodialysis trials: a systematic review. A J Kidney Dis. 2018،; 71:382-391. doi: 10.1053/j.ajkd.2017.09.018.

4. National Institutes of Health, National Institute of Diabetes and Digestive and Kidney Diseases. Annual Data Report: Atlas of End-Stage Renal Disease in the United States, Bethesda: US Renal Data System, USRDS; 2005.

5. Foley RN, Guo H, Snyder JJ, Gilbertson DT, Collins AJ. Septicemia in the United States dialysis population 1991 to 1999. J Am Soc Nephrol. 2004;15:1038-45. doi: 10.1097/01asn.0000119144.95922.c4.

6. James MT, Laupland KB, Tonelli M, Manns BJ, Culleton BF, Hemmelgarn BR. Risk of bloodstream infection in patients with chronic kidney disease not treated with dialysis. Arch Intern Med. 2008;168:2333-9. doi: 10.1001/archinte.168.21.2333.

7. Reynvoet E, Vandijck DM, Blot SI, Dhondt AW, De Waele JJ, Claus $S$, et al. Epidemiology of infection in critically ill patients with acute renal failure. Crit Care Med. 2009;37:2203-9. doi: 10.1097/CCM.0b013e3181a03961.

8. Girndt M, Sester M, Sester U, Kaul H, Kohler H. Molecular aspects of T- and B-cell function in uremia. Kidney Int Suppl. 2001;78:S206-211. doi: 10.1046/j.15231755.2001.59780206x.

9. James MT, Laupland KB, Tonelli M, Manns BJ, Culleton BF, Hemmelgarn BR. CKD and risk of hospitalization and death with pneumonia. Am J Kidney Dis 2009;54:24-32. doi: 10.1053/j.ajkd.2009.04.005.

10. Bossink A, Groeneveld J, Lambertus G. Prediction of microbial infection and mortality in medical patients with fever: plasma procalcitonin, neutrophilic elastase-alpha-antitrypsin and lactoferrin compared with clinical variables. Clin Infect Dis.1999;29:398-407. doi: 1086/520222.

11. Lu XL, Xiao ZH, Yang MY, Zhu YM. Diagnostic value of serum procalcitonin in patients with chronic renal insufficiency: a systematic review and meta-analysis. Nephrol Dial Transplant. 2012;28:122-9. doi:10.1093/ndt/gfs339.

12. Christ-Crain $M$, Müller B. Biomarkers in respiratory tract infections: diagnostic guides to antibiotic prescription, prognostic markers and mediators. Eur Respir J. 2007;30:55673. doi: 10.1183/09031936.00166106.

13. Fauci AS, Morens DM. The perpetual challenge of infectious diseases. N Engl J Med. 2012;366:454-61. doi: 10.1056/ NEJMra1108296.

14. Reynvoet E, Vandijck DM, Blot SI, Dhondt AW, De Waele JJ, Claus $S$, et al. Epidemiology of infection in critically ill patients with acute renal failure. Crit Care Med. 2009;37(7):2203-9. doi: 10.1097/CCM.0b013e3181a03961.

15. Shafazand $S$, Weinacker AB Blood cultures in the critical care unit: Improving utilization and yield. Chest. 2002;122:17271736. doi:10.1387/chest.122.5.1727.

16. Bressan S, Andreola B, Zucchetta P, Montini G, Burei M, Perilongo G, Da Dalt L. Procalcitonin as a predictor of renal scarring in infants and young children. Prediatr Nephrol 2009; 24: 1199-220.doi: 10.1007/s00467-009-1125-x.

17. Assicot M, Gendrel D. Carsin H, Raymond J, Guilbaud J, 
Bohuon C. High serum procalcitonin in patients with sepsis and infection. Lancet 1993;341:515-518. doi:10.1016/01406736(93)90277-n.

18. Pfafflin A, Schleicher E. Inflammation markers in point of-care testing (POCT). Anal Bioanal Chem 2009;393:1473-80. doi: 10.1007/s00216-008-2561-3.

19. Monneret G, Doche C, Duran DV, Lepape A, Bienvenu J. Procalcitonin as a specific marker of bacterial infection in adults. Clin Chem Lab Med. 1998;36:67-68

20. Le Moullec JM, Jullienne A, Chenais J, Lasmoles F, Guliana JM, Milhaud G, Oukhtar MS .The complete se-quence of human preprocalcitonin. FEBS. 1984;167:93-7. doi: 10.1016/00145793(84)80839-x.

21. Meisner M, Schmidt J, Huttner H, Tschaikowsky K. The natural elimination rate of procalcitonin in patients with normal and impaired renal function. Intensive Care Med J. 2000s26:S212-216. doi: 10.1007/BF02900740.

22. Torres A, Rodriguez M, Felsenfeld A, Martin-Malo A, Llach F. Sigmoidal relationship between calcitonin and calcium: studies in normal, parathyroidectomized, and azotemic rats. Kidney Int. 1991;40:700-4. doi: 10.1038/ki.1991.263.

23. Herget-Rosental S, Marggraf G, Pietruck F, Hüsing J, Strupat M, Philipp T, et al. Procalcitonin for accurate detection of infection in hemodialysis. Nephrol Dial Transplant. 2001;16:975-979. Doi:10.1093

24. Evenepoel P, Wol M. A balanced view of calcium and phosphate homeostasis in chronic kidney disease. Kidney Int. 2013;83:789-91. doi:10.1038/ki.2013.21.

25. Lindberg M, Hole A, Johnsen H, Asberg A, Rydning A, Myrvold $\mathrm{HE}$, et al. Reference intervals for procalcitonin and C-reactive protein after major abdominal surgery. Scand J Clin lab Invest. 2002;62:189-94 doi: 10.1080/003655102317475443.

26. Meisner M, Tschaikowsky K, Hutzler A, Schick C, Schuttler J. Postoperative plasma concentrations of procalcitonin after different types of surgery. Intensive Care Med. 1998;24:680-4. doi: $10.1007 / \mathrm{s} 001340050644$.
27. Oberhofer D, Rumenjak V, lazic J, Vucic N. Inflammatory indicators in patients after surgery of the large intestine. Acta Med Croatica. 2006;60:429-33.

28. Lee WS, Kang DW, Back JH, Kim HL, Chung JH, Shin BC. Cutoff value of serum procalcitonin as a diagnostic biomarker of infection in end-stage renal disease patients. Korean J Intern Med. 2015;30(2):198-204. doi: 10.3904/kjim.2015.30.2.198.

29. Simon L, Gauvin F, Amre DK, Saint-Louis P, Lacroix J: Serum procalcitonin and C-reactive protein levels as markers of bacterial infection: a systematic review and meta-analysis. Clin Infect Dis 2004, 39:206 217. doi: 10.1086/421997.

30. El-Sayed D, Grotts J, Golgert WA, Sugar AM. Sensitivity and specificity of procalcitonin in predicting bacterial infections in patients with renal impairment. Open Forum Infect Dis. 2014 21;1:68. doi:10.1093/ofid/ofu068.

31. Herget-Rosenthal S, Marggraf G, Pietruck F, Hüsing J, Strupat $M$, Philipp $T$, et al. Procalcitonin for accurate detection of infection in hemodialysis. Nephrol Dial Transplant. 2001;16:975-9. doi:10.1093/ndt/16.5.975.

32. Greg S Martin.Sepsis, severe sepsis and septic shock: changes in incidence, pathogens and outcomes. Expert Rev Anti Infect Ther. 2012;10:701-6. doi: 10.1586/eri.12.50.

33. Steinbach G1, Bölke E, Grünert A, Störck M, Orth K. Procalcitonin in patients with acute and chronic renal insufficiency. Wien Klin Wochenschr. 2004;116:849-53. doi: 10.1007/s00508-004-0279-6.

34. Dumea R1, Siriopol D, Hogas S, Mititiuc I, Covic A. Procalcitonin: diagnostic value in systemic infections in chronic kidney disease or renal transplant patients. Int Urol Nephrol. 2014; 46:461-8. doi: 10.1007/s11255-013-0542-8.

35. Fadel Fl, Elshamaa MF, Elghoroury EA, Badr AM, Kamel S, El-Sonbaty $M M$, et al. Usefulness of serum procalcitonin as a diagnostic biomarker of infection in children with chronic kidney disease. Arch Med Sci Atheroscler Dis. 2016;1(1):e23-e31. doi: 10.5114/amsad.2016.59672. 